\title{
HIV-Associated Malignancies
}

\author{
Kai Hübel
}

Department I of Internal Medicine, University Hospital Cologne, Cologne, Germany

The Acquired Immune Deficiency Syndrome (AIDS) pandemic was first reported in 1981 with the description of patients with opportunistic infections, pneumonia mainly caused by Pneumocystis jirovecii (formerly known as $P$. carinii), and cytomegalovirus infections [1]. It was quickly learned that patients infected with human immunodeficiency virus (HIV) also have a high risk of developing several forms of cancer. These malignancies may arise when the immune system is damaged and CD4 T-cell count is low. Virally induced neoplasia, such as Kaposi's sarcoma (associated with human herpes virus 8, HHV8), cervical cancer (associated with human papilloma virus, HPV), and aggressive non-Hodgkin's lymphoma (associated with Epstein-Barr virus, EBV) have been defined as AIDS-related cancers. However, with the advent of combination antiretroviral therapy (cART) in 1996 not only the risk of opportunistic infections declined but also the incidence of AIDSrelated cancers. Especially, the occurrence of Kaposi's sarcoma decreased dramatically. Furthermore, the introduction of cART broadens the spectrum of feasible chemotherapy protocols for the treating oncologist. As in HIV-negative patients, it became possible to use intensive regimens, including high-dose therapy followed by autologous stem cell transplantation.

Nevertheless, cancer remains a major cause of morbidity and mortality in HIV-infected individuals. It accounts for approximately one-third of all deaths in these patients. In fact, HIV patients have a higher risk to die from a tumor than from an AIDSdefined infection, with aggressive non-Hodgkin's lymphoma as the most frequent event. Therapy of these cancers remains a challenge for the treating oncologist. Besides detailed knowledge about the tumor itself and the therapeutic options, experience in the use of antiviral medication is essential.

Several guidelines and expert consensus reviews have already been published for HIV-infected patients with cancer, e.g. the British HIV association guideline for HIV-associated malignancies [2], the recommendations of the French CANCERVIH working group [3] or the German Onkopedia guidelines for HIV lymphoma [4]. The objective of this review series is to provide an update on current thera- peutic approaches for selected AIDS-related and non-AIDS-related malignancies and to describe possible drug-drug interactions.

Brunnberg et al. [5] focus on HIV-associated lymphomas. Despite the use of intensive protocols in the cART era, the outcome of non-Hodgkin's lymphoma is still worse compared to HIV-negative patients, and the optimal treatment strategy has not been defined yet. The authors also summarize data for HIV-associated Hodgkin's lymphoma, which shows a 10 - to 20 -fold higher incidence compared to the HIV-negative population, despite optimized anti-infective treatments. Kiderlen and colleagues [6] present date on lung cancer and HIV. The high prevalence of smoking in the HIV-infected population increases the risk of lung cancer, which is often diagnosed in more advanced stages and in younger patients compared to immunocompetent individuals. Hoffmann et al. [7] highlight the specifics in the treatment of Kaposi's sarcoma, the crucial role of consequent cART, and new therapeutic options. Oette et al. [8] discuss recent approaches to anal cancer, which has an incidence up to 40 times higher compared with the general population. Jensen et al. [9] describe individualized treatment options for HIV-associated gastrointestinal carcinoma, including liver cancer. Hentrich and Pfister [10] review data on urogenital tumors. Finally, Welz et al. [11] discuss important drug interactions which may not only increase toxicities but also may influence the overall prognosis of the patient.

In summary, the close cooperation of oncologists and infectious disease experts is mandatory for a successful management of HIVpositive individuals with cancer. In the cART era, treatment options are getting close to those of HIV-negative patients; however, the overall outcome is still worse in most malignancies. Furthermore, there is no defined standard treatment for HIV-associated cancer. The delineation of the most effective but least toxic treatment for an HIV patient with cancer will be a major objective of future clinical trials.

\section{Disclosure Statement}

The author declares no conflicts of interest.

\section{KARGER}

(๑) 2017 S. Karger GmbH, Freiburg

Fax +497614520714
Prof. Dr. med. Kai Hübel 


\section{References}

1 Centers for Disease Control (CDC): Pneumocystis pneumonia - Los Angeles. MMWR Morb Mortal Wkly Rep 1981:30:250-252.

2 British HIV Association guidelines for HIV-associated malignancies: HIV Med 2014;15(Suppl 2).

3 Spano JP, Poizot-Martin I, Costagliola D, et al.: NonAIDS-related malignancies: expert consensus review and practical applications from the multidisciplinary CANCERVIH Working Group. Ann Oncol 2016;27: 397-408.
4 Hentrich M, Hensel M, Hoffmann C, et al.: HIV-associated lymphomas. Onkopedia-Leitlinien, DGHO 2014. https://www.onkopedia.com/de/onkopedia/guidelines/hiv-assoziierte-lymphome.

5 Brunnberg U, Hentrich M, Hoffmann C, Wolf T, Hübel KH: HIV-associated malignant lymphoma. Oncol Res Treat 2017;40 DOI: 10.1159/000456036.

6 Kiderlen TR, Siehl J, Hentrich M: HIV-associated lung cancer. Oncol Res Treat 2017;40 DOI: 10.1159/ 000458442.

7 Hoffmann C, Sabranski M, Esser S: HIV-associated Kaposi's sarcoma. Oncol Res Treat 2017;40 DOI: $10.1159 / 000455971$.
8 Oette M, Mosthaf F, Sautter-Bihl M, Esser S: HIV-associated anal dysplasia and anal carcinoma. Oncol Res Treat 2017;40 DOI: 10.1159/000456715.

$\checkmark$ Jensen BEO, Oette M, Haes J, Häussinger D: HIV-associated gastrointestinal cancer. Oncol Res Treat 2017; 40 DOI: $10.1159 / 000456714$.

10 Hentrich M, Pfister D: HIV-associated urogenital malignancies. Oncol Res Treat 2017;40 DOI: 10.1159/ 000457130.

11 Welz T, Wyen C, Hensel M: Drug interactions in the treatment of malignancy in HIV-infected patients. Oncol Res Treat 2017;40 DOI: 10.1159/000458443. 